\title{
Vector Velocity Estimation for Portable Ultrasound using Directional Transverse Oscillation and Synthetic Aperture Sequential Beamforming
}

di lanni, Tommaso; Hemmsen, Martin Christian; Jensen, Jørgen Arendt

Published in:

Proceedings of 2016 IEEE International Ultrasonics Symposium

Link to article, DOI:

10.1109/ULTSYM.2016.7728777

Publication date:

2016

Document Version

Peer reviewed version

Link back to DTU Orbit

Citation (APA):

di lanni, T., Hemmsen, M. C., \& Jensen, J. A. (2016). Vector Velocity Estimation for Portable Ultrasound using Directional Transverse Oscillation and Synthetic Aperture Sequential Beamforming. In Proceedings of 2016 IEEE International Ultrasonics Symposium IEEE. https://doi.org/10.1109/ULTSYM.2016.7728777

\section{General rights}

Copyright and moral rights for the publications made accessible in the public portal are retained by the authors and/or other copyright owners and it is a condition of accessing publications that users recognise and abide by the legal requirements associated with these rights.

- Users may download and print one copy of any publication from the public portal for the purpose of private study or research.

- You may not further distribute the material or use it for any profit-making activity or commercial gain

- You may freely distribute the URL identifying the publication in the public portal 


\title{
Vector Velocity Estimation for Portable Ultrasound using Directional Transverse Oscillation and Synthetic Aperture Sequential Beamforming
}

\author{
Tommaso Di Ianni, Martin Christian Hemmsen, Jørgen Arendt Jensen \\ Center for Fast Ultrasound Imaging, Department of Electrical Engineering, \\ Technical University of Denmark, DK-2800 Lyngby, Denmark
}

\begin{abstract}
In this paper, a vector flow imaging method is presented, which combines the directional transverse oscillation approach with synthetic aperture sequential beamforming to achieve an efficient estimation of the velocities. A doubleoscillating field is synthesized using two sets of focused emissions separated by a distance in the lateral direction. A lowresolution line (LRL) is created for each emission in the first stage beamformer, and a second beamformer provides the highresolution data used for the velocity estimation. The method makes it possible to have continuously available data in the whole image. Therefore, high and low velocities can be estimated with a high frame rate and a low standard deviation. The first stage is a fixed-focus beamformer that can be integrated in the transducer handle, enabling the wireless transmission of the LRLs. The approach does not require any angle compensation or prior knowledge on the beam-to-flow angle. The feasibility of the method is demonstrated through simulations and flow rig measurements of a parabolic flow in a vessel at 90-degree beam-to-flow angle. The mean bias obtained from 50 independent measurements is equal to $\mathbf{- 0 . 6 7 \%}$ for the lateral profile and $0.43 \%$ for the axial profile. The relative standard deviation is $3.19 \%$ and $0.47 \%$ for the lateral and axial profiles. It is, therefore, demonstrated that vector velocity estimation can be efficiently integrated in a portable ultrasound scanner with state-of-the-art performance.
\end{abstract}

\section{INTRODUCTION}

The visualization of blood flow dynamics in a vectorial form is an effective tool for the assessment of a number of cardiovascular diseases. The estimation of 2-D velocity vectors allows the clinicians to extract useful information from complex flow patterns and perform quantitative measurements. A number of vector flow imaging (VFI) methods have been proposed in the literature [1]-[4]. Recently, Jensen [5] presented a directional transverse oscillation (DTO) approach, which improves the previous transverse oscillation (TO) implementation.

The integration of VFI in a handheld scanner opens a wide range of new potential applications. The feasibility of a color flow mapping sequence implemented in a consumerlevel tablet was demonstrated by Hemmsen et al. [6] based on synthetic aperture sequential beamforming (SASB) [7], [8]. The fixed-focused ultrasound lines were wirelessly transmitted to the tablet, where re-focusing and velocity estimation were performed. The approach was based on directional beamforming [9]. Due to the high computational requirements of the method, given, in particular, by the necessity of crosscorrelating the directional lines for the velocity estimation, thermal issues compromised the real-time performance of the imaging sequence. Furthermore, the method requires the knowledge of the flow angle, which needs to be estimated with increased computational demand.

In this paper, a VFI method is presented, which combines SASB with DTO to achieve a more efficient estimation of the velocities. The method is based on an autocorrelation approach, and does not require any prior estimation of the flow angle. Simulations of a parabolic flow profile in a vessel at $90^{\circ}$ beam-to-flow angle were performed in Field II [10], [11] using a linear array transducer to demonstrate the feasibility of the approach. Measurements in a flow rig, finally, demonstrate its performance in a realistic situation.

\section{BACKGROUND AND THEORY}

\section{A. Directional Transverse Oscillation}

In DTO, a weakly focused beam is emitted and the ultrasound field received by all the transducer elements is beamformed using two apodized apertures separated by a distance in the lateral direction. This creates a double-oscillating pulseecho field, which allows to estimate the blood velocity in the axial and lateral dimensions.

At a given depth $l$, the signal $x(k, l, m)$ is beamformed in the direction transverse to the direction of propagation of the ultrasound beam, with $k$ the sample index along the lateral direction, and $m$ the emission index. The quadrature signal required for the velocity estimation [12] is then obtained by performing a Hilbert transform of the lateral signal

$$
y(k, l, m)=\mathcal{H}_{k}\{x(k, l, m)\},
$$

where $\mathcal{H}_{k}$ is the Hilbert transform in the direction of $k$.

A complex signal

$$
r_{s q}(k, l, m)=x(k, l, m)+j y(k, l, m)
$$

is created by combining the in-phase and quadrature lateral signals, and the lateral wavelength is calculated as:

$$
\frac{1}{\lambda_{x}(l, m)}=f_{x}(l, m)=\frac{\sum_{f=-N / 2}^{N / 2} \frac{f}{N \Delta k}\left|R_{s q}(f, l, m)\right|^{2}}{\sum_{f=-N / 2}^{N / 2}\left|R_{s q}(f, l, m)\right|^{2}}
$$




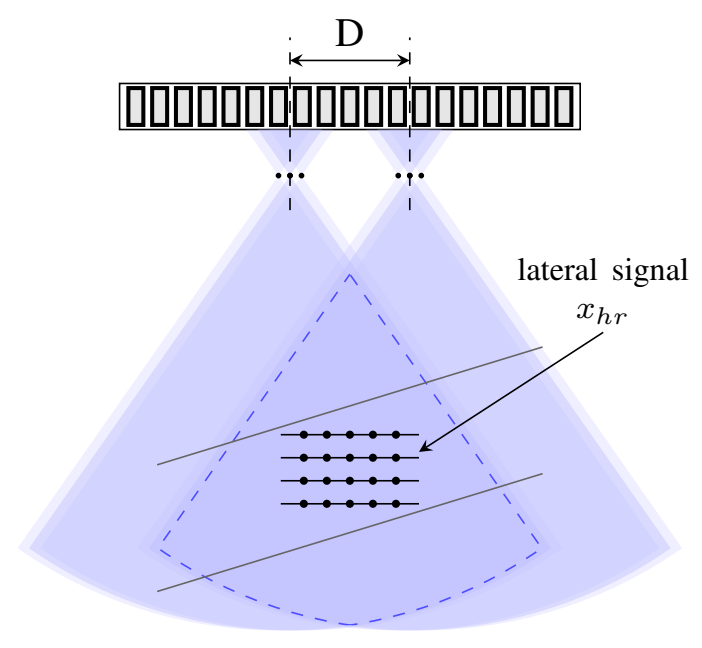

Fig. 1. Imaging setup for SASB DTO with a linear array transducer.

with $R_{s q}$ the Fourier transform of the directional signal $r_{s q}$ along the lateral direction, $N$ the number of Fourier coefficients, $f$ the sample index in the Fourier domain, and $\Delta k$ the lateral spatial sampling period. For the equations involved in the velocity estimation, readers are referred to [5].

Contrary to conventional TO, the lateral oscillation wavelength $\lambda_{x}$ is directly estimated as a function of the depth from the beamformed data, relieving the method of the need to precisely calibrate the beamformers. $\lambda_{x}$ can also be averaged over the axial dimension to improve the variance of the estimate. Furthermore, DTO decouples the lateral wavelength from the apodization function, maintaining a low bias for the velocity estimation along the depth.

\section{B. Laterally oscillating field using SASB}

In the proposed method, the laterally oscillating field is synthesized using two sets of virtual sources (VSs) separated by a distance $D$, as shown in Fig. 1. Each set is referred to as virtual aperture (VA). The VSs are obtained by using a static focus in transmit and receive. For each emission, a low-resolution line (LRL) is beamformed in the first stage. A double-oscillating field is then created by the second stage beamformer from a set of $K$ weighted LRLs - $K=6$ in Fig. 1 - in the whole high-resolution image (HRI) at once (dashed line in Fig. 1). The extent of this image is a function of the f-number of the VSs and the distance $D$.

The high-resolution lateral signal $x_{h r}(k, l, m)$ is found at the depth $l$ in the $m$-th HRI. The velocity estimation is performed as explained in Sec. II-A, where $x_{h r}$ is used in place of $x$ in (1) and (2). A new lateral signal is calculated after every $K$ emissions. Therefore, the effective pulse repetition period for the flow estimation, referred to as $T_{p r f}^{e f f}$, is equal to $K T_{p r f}$, with $T_{p r f}$ the repetition period of the flow sequence.

It must be noticed that, unlike DTO, which estimates the velocities on a line base, the proposed approach provides velocity estimates everywhere in the HRI. Therefore, continuous
TABLE I

\begin{tabular}{lcc}
\multicolumn{3}{c}{ FIXED SIMULATION AND MEASUREMENT PARAMETERS } \\
\hline Speed of sound & 1490 & $\mathrm{~m} \mathrm{~s}^{-1}$ \\
Transducer parameters & Linear array & - \\
Transducer & 0.55 & $\lambda$ \\
Element pitch & & \\
Virtual sources parameters & 4-cycle weighted & - \\
Excitation & 4.1 & $\mathrm{MHz}$ \\
Center frequency & Rectangular & - \\
Transmit apodization & Hamming & - \\
Receive apodization & 1.19 & - \\
f-number - f\# & 10 & $\mathrm{~mm}$ \\
Axial position & 6 & - \\
Number of VSs - K & 1.5 & $\mathrm{kHz}$ \\
Effective repetition frequency - $f_{p r f}^{e f f}$ & Rectangular & - \\
VA apodization & & - \\
Beamforming parameters & 10000 & $1 / \mathrm{m}$ \\
Lateral sampling frequency & 32 & $\mathrm{sample}$ \\
Lateral signal length & & \\
&
\end{tabular}

TABLE II

\begin{tabular}{ccc}
\multicolumn{3}{c}{ PARAMETERS VARIED IN SIMULATION } \\
\hline \multirow{2}{*}{ VA distance - $D$} & $16-24-32-40$ & \\
& $\mathbf{4 8}-56-64-72$ & element \\
HRI per estimate - $M$ & $8-16-\mathbf{3 2}$ & - \\
& $64-96-128$ & - \\
\hline
\end{tabular}

data is available, and high and low velocities can be estimated at once with a high frame rate and a low standard deviation. Furthermore, only one beamformed LRL for each emission needs to be transmitted from the probe to the processing unit, if the fixed-focus beamformer is implemented in the probe handle. As a result, the transmission can be achieved through a wireless link.

\section{METHODS}

\section{A. Simulation setup}

Simulations were performed in Field II to investigate the potential of the method. A simulation model was developed in MATLAB (The MathWorks Inc., Natick, MA, USA), and the second stage beamformer was implemented using the BFT3 toolbox [13]. The parameters fixed for all the simulations are reported in Table I. A $0.55 \lambda$-pitch linear array transducer was used with a center frequency of $4.1 \mathrm{MHz}$. The effective pulse repetition frequency for the flow sequence was set to $f_{p r f}^{e f f}=$ $1.5 \mathrm{kHz}\left(T_{p r f}^{e f f}=1 / f_{p r f}^{e f f}\right)$. The f-number was $f \#=1.19$.

A parabolic flow in a vessel with a $6-\mathrm{mm}$ radius located at a depth of $50 \mathrm{~mm}$ with $90^{\circ}$ beam-to-flow angle was simulated. At least 20 scatterers per resolution volume were considered to ensure fully developed speckle. The peak velocity was $v_{\max }=$ $0.2 \mathrm{~m} \mathrm{~s}^{-1}$. The distance $D$ between the VAs and the number $M$ of HRIs used per velocity estimate were varied to investigate their effect on the performance of the approach (Table II). The mean bias and the relative standard deviation for the velocity estimation were calculated inside the vessel from 50 independent simulations.

\section{B. Measurement setup}

A parabolic flow in a $6-\mathrm{mm}$ radius tube was scanned in a flow rig system. The SARUS scanner [14] was connected to a 

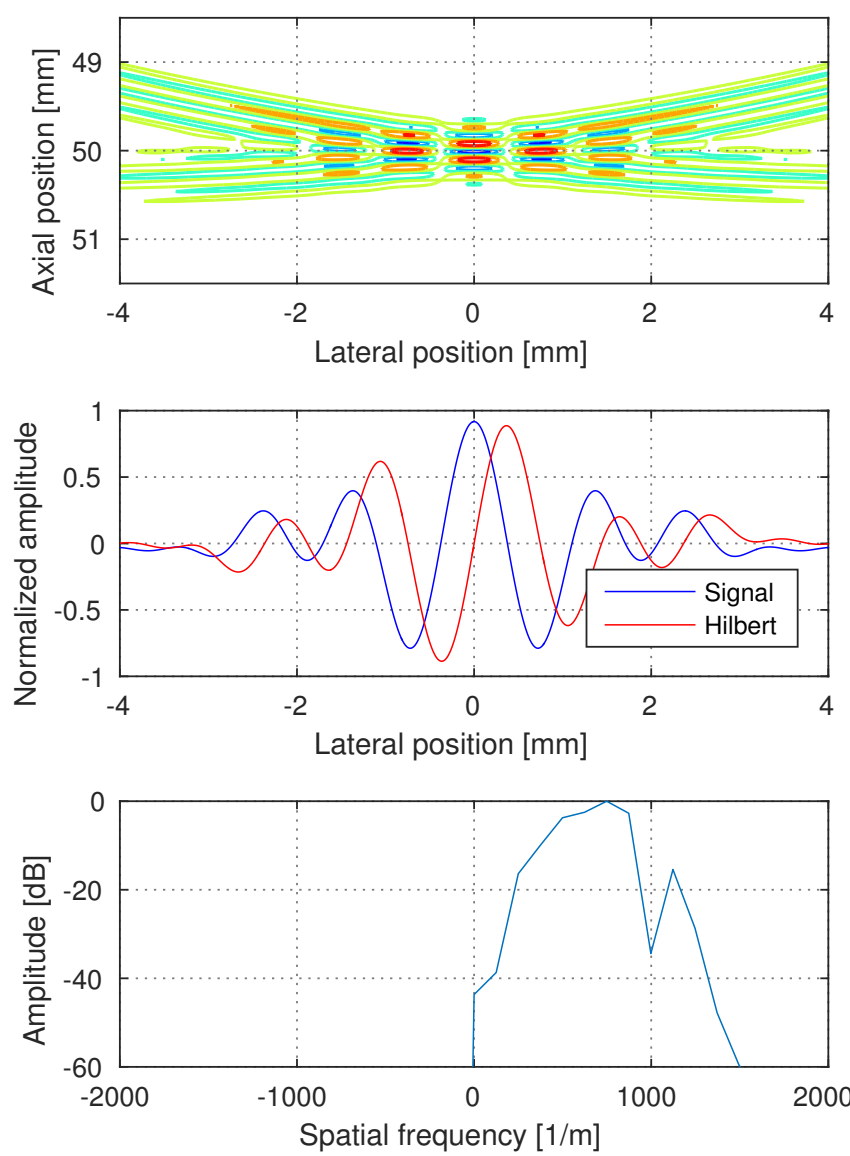

Fig. 2. The simulated double-oscillating PSF at $50 \mathrm{~mm}$ for $D=48$ is shown in the top figure; the signal obtained by sampling the PSF at the peak point in the lateral direction and its Hilbert transform are shown in the middle image; the bottom image shows the one-sided spectrum used for the estimation of the lateral wavelength.

BK Ultrasound (Herlev, Denmark) 2L8 linear array transducer, which has a center frequency of $4.1 \mathrm{MHz}$ and a $0.55 \lambda$ pitch. The volume flow was measured for reference using a magnetic flow meter (MAG3000, Danfoss, Nordborg, Denmark). The vessel was located at a depth of $50 \mathrm{~mm}$ with a beam-to-flow angle of $90^{\circ}$ and a peak velocity of $0.21 \mathrm{~m} \mathrm{~s}^{-1}$. The same parameters as in Table I were used for the measurements. The distance between the VAs was varied as in Table II, while $M$ was fixed to 32. The pulse repetition frequency of the system was set to $f_{p r f}=9 \mathrm{kHz}$. Stationary-echo cancelling was achieved by averaging 16 high-resolution images. The mean bias and the relative standard deviation were calculated for the points inside the vessel from 50 estimations with independent data.

\section{RESUlts}

The simulated double-oscillating point spread function (PSF) at a depth of $50 \mathrm{~mm}$ is displayed in Fig. 2 (top) for $D=48$, along with the signal obtained by sampling the PSF at the peak point in the lateral direction and its Hilbert transform (middle). The bottom figure shows the one-sided spectrum
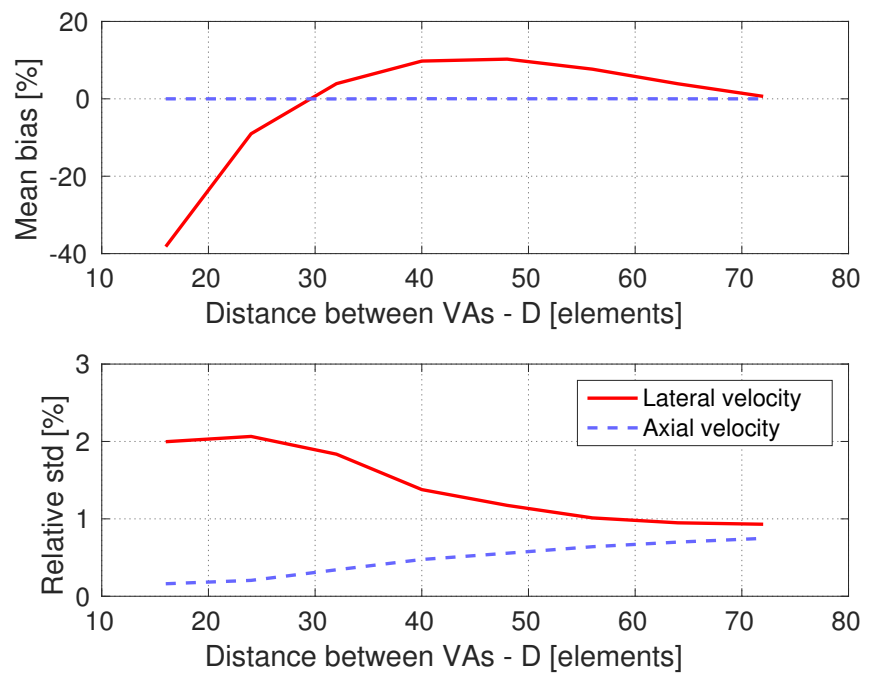

Fig. 3. Mean bias (top) and relative standard deviation (bottom) for the simulated lateral (red-solid) and axial (blue-dotted) velocity profiles as a function of the distance $D$ between VAs.
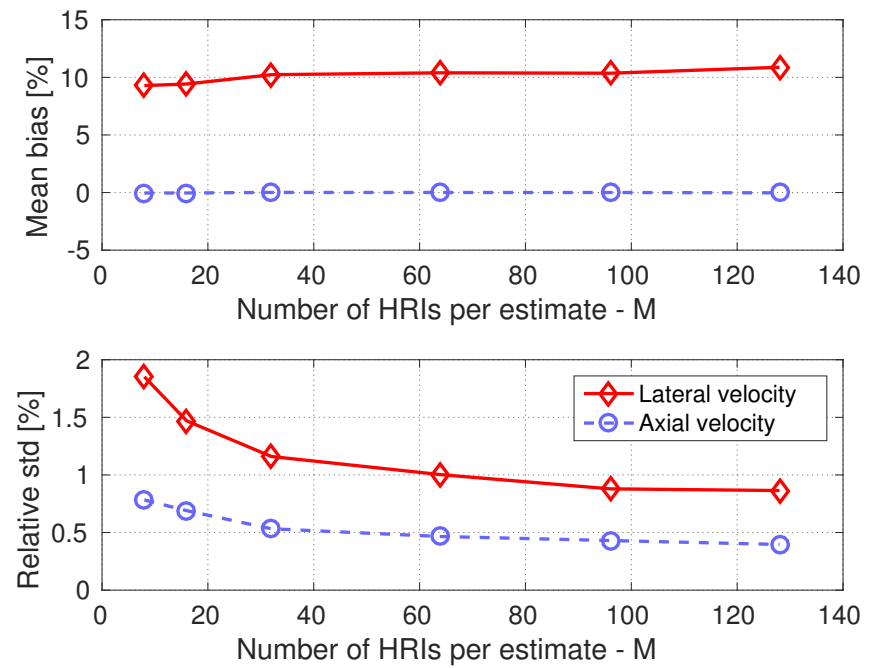

Fig. 4. Mean bias (top) and relative standard deviation (bottom) for the simulated lateral and axial profiles as a function of the number $M$ of HRIs used per estimate.

obtained by Fourier transforming the complex lateral signal. This is used to estimate the lateral wavelength as in (3).

Fig. 3 shows the mean bias (top) and the relative standard deviation (bottom) for the simulated velocity profiles as a function of the distance $D$. The mean bias for the lateral profile decreases with increasing $D$, and the minimum value for this setup is found for $D=72$, and is equal to $-0.64 \%$. The distance $D$ can be thought of as the aperture width of the DTO system, and has a significant impact on the lateral oscillation wavelength. Larger distances provide shorter wavelengths, hence a higher lateral resolution, at the expenses of a slightly reduced image.

The mean bias and the relative standard deviation as a function of the number $M$ of HRIs used per estimate are 



Fig. 5. Mean bias (top) and relative standard deviation (bottom) for the measured lateral (red-solid) and axial (blue-dotted) velocity profiles as a function of the distance $D$ between VAs.

shown in Fig. 4 for simulated data. The standard deviation is, in particular, decreased by increasing $M$, and is equal to $0.86 \%$ and $0.4 \%$ for the lateral and axial profiles with $M=128$.

Fig. 5 shows the mean bias (top) and the relative standard deviation (bottom) for the measured velocity profiles as a function of the distance $D$. A significant mismatch is noticed in the mean bias for the measured lateral profile compared with the simulated one. In particular, a minimum is found for $D=24$, which is equal to $-0.67 \%$, and the curve diverges for increasing values of $D$. Further research is needed to investigate the reasons of this inconsistency.

The measured lateral (top) and axial (bottom) velocity profiles for $D=24$ are displayed in Fig. 6. The green curves show the reference profiles calculated from the measured volume flow, the blue curves are the mean profiles from 50 independent estimations, and the red curves show +/-3 standard deviations. The mean bias is equal to $-0.67 \%$ for the lateral profile and $-0.43 \%$ for the axial profile. The mean relative standard deviation is $3.19 \%$ for the lateral profile and $0.47 \%$ for the axial profile.

\section{CONCLUSION}

This paper presented a VFI method, which combines DTO and SASB to achieve an efficient velocity estimation. The method makes it possible to have continuous data acquisition, i.e. data used for the estimation is continuously available everywhere in the HRI. This allows for a high frame rate and a low standard deviation, and simplifies the implementation of the stationary-echo filter. Furthermore, if the first stage beamformer is integrated in the probe handle, wireless transmission of the ultrasound data to the processing unit can be achieved. The approach also enables the possibility of implementing synthetic aperture VFI in cart-based commercial scanners, owing to the lower computational complexity compared with previous implementations.
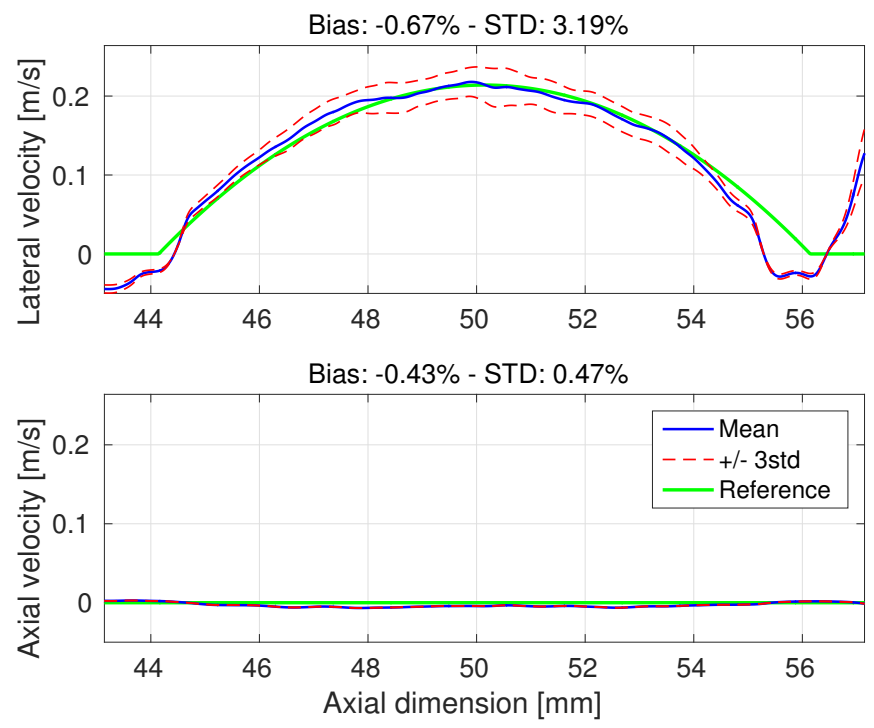

Fig. 6. Lateral (top) and axial (bottom) velocity profiles measured in the flow rig at $90^{\circ}$ beam-to-flow angle.

\section{REFERENCES}

[1] M. D. Fox, "Multiple crossed-beam ultrasound Doppler velocimetry," IEEE Trans. Son. Ultrason., vol. SU-25, pp. 281-286, 1978.

[2] G. E. Trahey, J. W. Allison, and O. T. von Ramm, "Angle independent ultrasonic detection of blood flow," IEEE Trans. Biomed. Eng., vol. BME-34, pp. 965-967, 1987.

[3] J. A. Jensen and P. Munk, "A new method for estimation of velocity vectors," IEEE Trans. Ultrason., Ferroelec., Freq. Contr, vol. 45, pp. 837-851, 1998.

[4] M. E. Anderson, "Multi-dimensional velocity estimation with ultrasound using spatial quadrature," IEEE Trans. Ultrason., Ferroelec., Freq. Contr., vol. 45, pp. 852-861, 1998.

[5] J. A. Jensen, "Improved vector velocity estimation using directional transverse oscillation," in Proc. IEEE Ultrason. Symp. IEEE, 2015, pp. $1-4$.

[6] M. C. Hemmsen, L. Lassen, T. Kjeldsen, J. Mosegaard, and J. A. Jensen, "Implementation of real-time duplex synthetic aperture ultrasonography," in Proc. IEEE Ultrason. Symp., 2015, pp. 1-4.

[7] J. Kortbek, J. A. Jensen, and K. L. Gammelmark, "Sequential beamforming for synthetic aperture imaging," Ultrasonics, vol. 53, no. 1, pp. $1-16,2013$.

[8] M. C. Hemmsen, P. M. Hansen, T. Lange, J. M. Hansen, K. L. Hansen, M. B. Nielsen, and J. A. Jensen, "In vivo evaluation of synthetic aperture sequential beamforming," Ultrasound Med. Biol., vol. 38, no. 4, pp. 708716, 2012.

[9] J. A. Jensen and S. I. Nikolov, "Directional synthetic aperture flow imaging," IEEE Trans. Ultrason., Ferroelec., Freq. Contr., vol. 51, pp. 1107-1118, 2004.

[10] J. A. Jensen and N. B. Svendsen, "Calculation of pressure fields from arbitrarily shaped, apodized, and excited ultrasound transducers," IEEE Trans. Ultrason., Ferroelec., Freq. Contr., vol. 39, pp. 262-267, 1992.

[11] J. A. Jensen, "Field: A program for simulating ultrasound systems," Med. Biol. Eng. Comp., vol. 10th Nordic-Baltic Conference on Biomedical Imaging, Vol. 4, Supplement 1, Part 1, pp. 351-353, 1996.

[12] J. A. Jensen, "A new estimator for vector velocity estimation," IEEE Trans. Ultrason., Ferroelec., Freq. Contr., vol. 48, no. 4, pp. 886-894, 2001.

[13] J. M. Hansen, M. C. Hemmsen, and J. A. Jensen, "An object-oriented multi-threaded software beamformation toolbox," in Proc. SPIE Med. Imag., vol. 7968, March 2011, pp. 79 680Y-1-79680Y-9.

[14] J. A. Jensen, H. Holten-Lund, R. T. Nilsson, M. Hansen, U. D. Larsen, R. P. Domsten, B. G. Tomov, M. B. Stuart, S. I. Nikolov, M. J. Pihl, Y. Du, J. H. Rasmussen, and M. F. Rasmussen, "Sarus: A synthetic aperture real-time ultrasound system," IEEE Trans. Ultrason., Ferroelec., Freq. Contr., vol. 60, no. 9, pp. 1838-1852, September 2013. 